\title{
Mobile EMR Use for Epidemiological Surveillance on a Medical Service Trip in Honduras: A Pilot Study
}

\author{
Christopher J. Dainton', Charlene H. Chu' ${ }^{2}$ \\ ${ }^{1}$ Grand River Hospital, Kitchener, Canada \\ ${ }^{2}$ University of Toronto, Toronto, Canada \\ Email: christopher.dainton@gmail.com
}

Received 30 December 2015; accepted 29 February 2016; published 4 March 2016

Copyright (C) 2016 by authors and Scientific Research Publishing Inc.

This work is licensed under the Creative Commons Attribution International License (CC BY). http://creativecommons.org/licenses/by/4.0/

c) (i) Open Access

\section{Abstract}

Rationale: Medical treatment on short-term primary care medical service trips (MSTs) is generally symptom-based and supplemented by point-of-care testing. This pilot study contributes to the effective planning for such austere settings based on predicted symptomology. Objective: We aimed to prospectively document the epidemiology of patients seen during two low-resource clinics on a MST in Honduras and apply predefined case definitions adapted from guidelines used by international healthcare organizations (e.g. World Health Organization). Methods: An observational design was used to track the epidemiology during two clinics on an MST in Limon, Honduras in March 2015. The QuickChart mobile electronic medical record (EMR) application was piloted to document diagnoses according to predefined case definitions. Results: The most commonly diagnosed syndromes were upper respiratory complaints $(20.19 \%)$, nonspecific abdominal complaints $(20.19 \%)$, general pain $(15.38 \%)$, hypertension $(9.62 \%)$, pruritus $(6.73 \%)$, and asthma/ COPD (4.81\%). The case definitions accounted for $94 \%$ of all complaints and diagnoses on the brigade. Discussion: The distribution of common patient diagnoses on this MST was similar to that which had been reported elsewhere. The use of broader symptom-based case definitions for epidemiologic surveillance could also facilitate the syndromic management of patients seen on MSTs, and improve the consistency of treatment offered. Conclusion: Case definitions for common syndromes on primary care MSTs may be a feasible method of standardizing patient management. Preliminary use of the QuickChart EMR was acceptable for documentation of epidemiology in the field. Further study is necessary to investigate the reliability of syndromic diagnostic criteria between different clinicians and in a variety of MST settings.

\section{Keywords}

Electronic Medical Records, Epidemiology, Global Health Experience, Medical Missions, Medical 


\section{Service Trip, Primary Care}

\section{Introduction}

Primary care medical service trips (MSTs) to low and middle income countries (LMICs) are common experiences for clinicians from high income countries (HICs), but quantitative literature describing the phenomenon remains in its infancy [1]. MSTs often take the form of mobile medical clinics providing primary care services to disenfranchised communities [2], often conducting general health assessments, preventive care, health promotion and education, and management of acute and chronic conditions. A key concern for non-governmental organizations (NGOs) in planning such trips is understanding the local epidemiology, which would inform appropriate resource allocation (e.g. medication formulary, clinical supplies) for each trip.

The primary barriers to high quality patient treatment are twofold. First, clinicians from HICs have a variety of professional backgrounds and may experience critical challenges to effective diagnosis and management during MSTs (e.g. limited expertise in tropical medicine, language barriers when communicating with the local patient population) [2]. Second, limited access to confirmatory microbiological, laboratory, and imaging tests means that clinicians must rely on syndromic diagnosis, supplemented by simple point-of-care tests (urine dip, urine pregnancy test, glucometer, hemoglobinometer) which can lack specificity and sensitivity. The lack of any clear diagnostic criteria may contribute to high variability in the treatment choices of clinicians and inaccurate descriptions of epidemiology, both of which jeopardize quality of care [3] [4].

As investments and interest in MSTs continue to grow [1], this demands a critical reflection on how clinician diagnoses are being documented and analyzed from an epidemiological perspective. Recent retrospective studies have described common diagnoses on mobile brigades [5], and suggested that the proportion of MST patients with selected diagnoses may be similar across multiple rural settings in Latin America [6] (Ecuador, Dominican Republic, and Guatemala), but such descriptions do not clarify the underlying diagnostic criteria used by the treating clinicians. In order to address the challenges of high diagnostic variability and potential inaccuracy of epidemiological surveillance, the application of broad, symptom based case definitions may be an appropriate solution. The use of case definitions could also facilitate the syndromic management of patients seen on MSTs, in order to improve the consistency of treatment offered.

Medical record keeping is variable in MST settings, and paper-based charting is often undermined by illegible handwriting [7], degradation of records by humid climates, and difficulties in transporting, accessing, and managing data [8] [9]. The logistical challenges of transporting MST records from a village clinic in Limon, Honduras to a satellite community (Francia, Honduras) are illustrated in Figure 1. Such trips may be a promising frontier for mobile electronic medical records (EMRs), allowing point of care documentation of patient records that may then inform allocation of resources. While there have been recent attempts to adapt them to austere practice environments [10] [11], we are unaware of any prior study that has used a mobile EMR to document epidemiologic case definitions on an MST.

The purpose of this observational study was to pilot a novel smart phone-based EMR app (QuickChart EMR), in order to prospectively document epidemiology according to predefined case definitions during two MST clinics in Limon, Honduras.

\section{Methods}

Three physicians (including the investigator), one physician assistant, and one nurse practitioner from the United States and Canada traveled to Limon, Honduras with Carolina Honduras Health on March 20-28, 2015. The clinicians were accompanied by four registered nurses, one pharmacist, and non-medical support staff. Limon is a remote Garifuno community in the coastal Atlantida province, with a population of approximately 8600 .

Clinics took place in an established clinic within the village on Day 1, and in a church in the peripheral community of Piedra Blanca on Day 2. A glucometer, hemoglobinometer, urine dipsticks, urine pregnancy tests, pulse oximeter, and rapid malaria test were immediately available for diagnostic purposes. Standard charting was performed on paper. A total of 116 patients were seen on Day 1 and 130 patients on Day 2. 


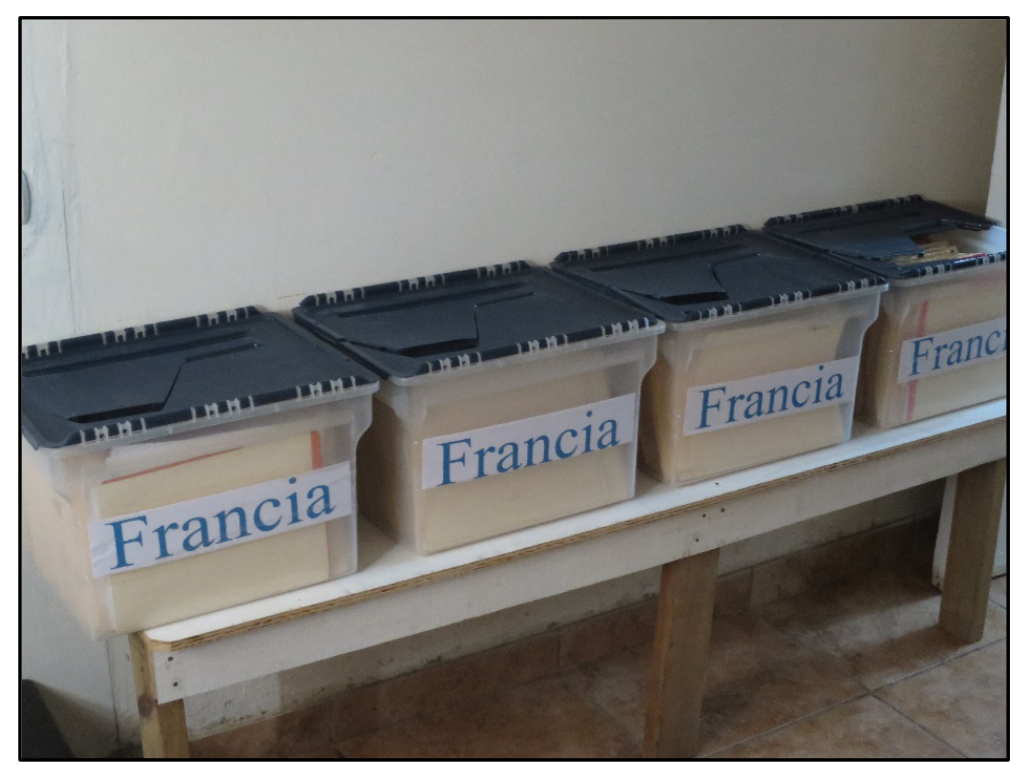

Figure 1. Medical records are transported in boxes for mobile clinics in peripheral communities like Francia (near Limon, Honduras).

\section{QuickChart EMR}

QuickChart EMR is a novel mobile application which supports clinical care with geographically relevant evidence based clinical guidelines and bilingual support. The app allows tracking of patients and diagnoses, secure production of medical notes and clinic reports in a pdf file, as well as epidemiologic analysis through export to an Excel file. The investigator charted primary diagnoses, age, and gender using the application on a Nexus 5 smartphone (Figure 2). Case definitions used in syndromic diagnosis were immediately available to the investigator through the decision support menu within the app.

Patient diagnoses were made by clinicians according to predefined case definitions. The specific case definitions were adapted and simplified based on World Health Organization (WHO) [12]-[14], Medicins sans Frontieres/Doctors Without Borders (MSF) [15] [16], and International Diabetes Federation (IDF) guidelines [17], as well as Dainton and Chu (2014) [3], and are described below in Table 1. Epidemiologic data was then extracted to an Excel file.

\section{Results}

A total of 246 patients were seen by all five providers during the first two clinic days. Fifty five patients were seen by the investigator, and their age ranges are described in Figure 3. The average age of patients assessed was 22 years.

Diagnoses are displayed in Figure 4. The mean number of diagnoses per patient was 1.9. The most common diagnoses were upper respiratory complaints (20.19\%), nonspecific abdominal complaints (20.19\%), general pain (15.38\%), hypertension (9.62\%), pruritus (6.73\%), and asthma/COPD (4.81\%).

Patient presentations not accounted for by the case definitions included aphthous ulcers, a resolved episode of epistaxis, a wound infection, and a complaint of poor vision. One patient complained of hand weakness secondary to an old gunshot wound to the shoulder, and already had a physiotherapist involved in his care. One patient arrived at the clinic with an acute exsanguinating hand laceration and flexor tendon injury, and he was sutured, splinted, and referred to an orthopedic team scheduled to arrive at the clinic the following week. Overall, the case definitions were able to account for $94 \%$ of all the patients who visited the clinics.

\section{Discussion}

This study builds on existing literature describing the symptomology that clinicians are likely to encounter on MSTs, and the epidemiology was similar [5]. General pain, upper respiratory complaints, pruritus and tinea, 

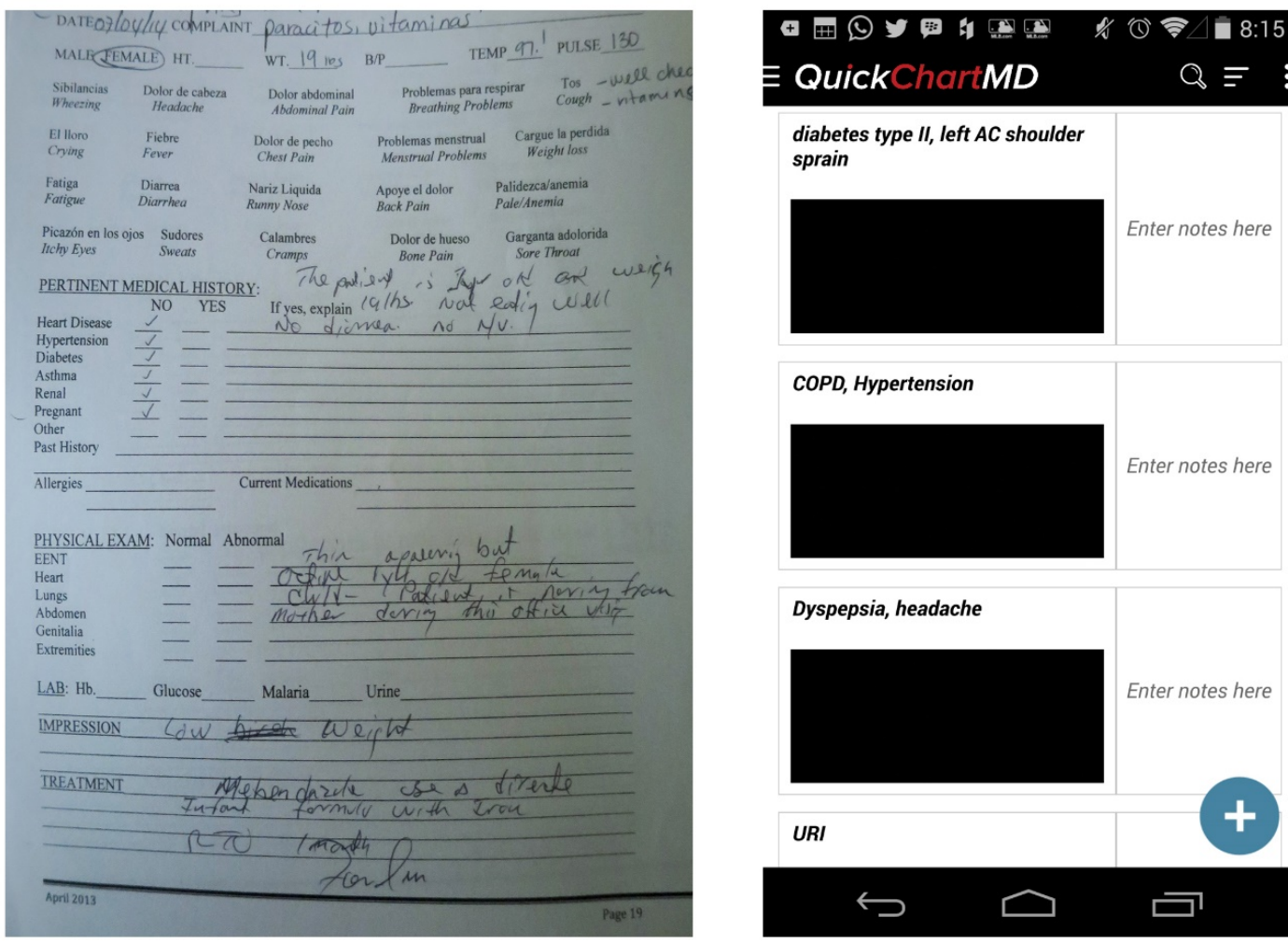

diabetes type II, left AC shoulder sprain

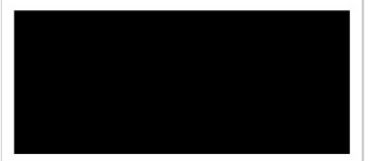

Enter notes here

COPD, Hypertension

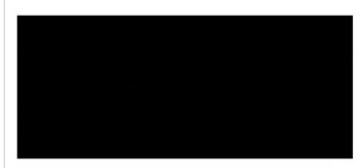

Enter notes here

Dyspepsia, headache

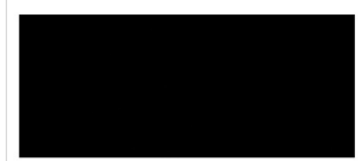

Enter notes here

URI

Figure 2. Comparison of a paper medical record (left) used on a primary care MST, with a screenshot of the QuickChart EMR application for Android (right, identifying patient information blacked out).

Table 1. Case definitions used to define common syndromes seen on a primary care MST in Limon, Honduras.

\begin{tabular}{|c|c|}
\hline Syndrome & Case definition \\
\hline General pain & Nonspecific headache, back pain, or myalgias worse after working, osteoarthritis, plantar fasciitis \\
\hline Hypertension [12] & Systolic BP $>140$ or diastolic BP $>90$ \\
\hline Diabetes $[18]$ & Random blood glucose $>11.1$ \\
\hline Upper respiratory complaints & URI, pharyngitis, or acute otitis media \\
\hline Asthma [13] & Childhood onset chronic intermittent cough, dyspnea, or wheeze \\
\hline COPD [17] & Persistent dyspnea or daily productive cough PLUS exposure to smoke, burning fossil fuels, or dust \\
\hline Dyspepsia [15] [16] & Epigastric discomfort with or without bloating, gas, or nausea \\
\hline Nonspecific abdominal complaints & $\begin{array}{l}\text { Poor appetite or lower abdominal discomfort without dyspepsia, pregnancy, diarrhea, } \\
\text { urinary symptoms, or vaginal discharge, OR parasites visualized in stool }\end{array}$ \\
\hline Vaginal discharge & Vaginal discharge or discomfort \\
\hline Menstrual complaints & Dysmenorrhea, menorrhagia, or abnormal uterine bleeding \\
\hline Pregnancy & Missed menses and positive pregnancy test \\
\hline Pruritis [14] & Itching involving two sites of body \\
\hline Tinea [14] & Single or multiple characteristic circular skin lesions \\
\hline
\end{tabular}




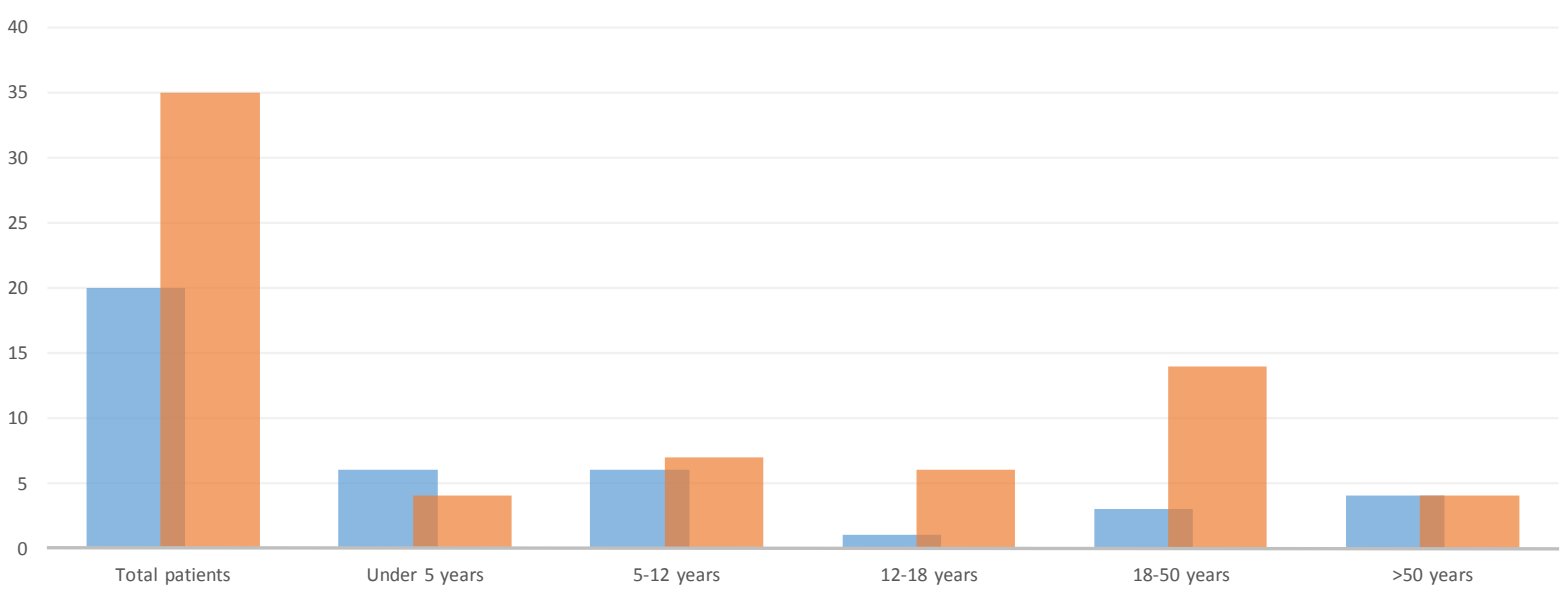

Figure 3. Age ranges and gender of patients assessed during two primary care brigade clinics near Limon, Honduras.

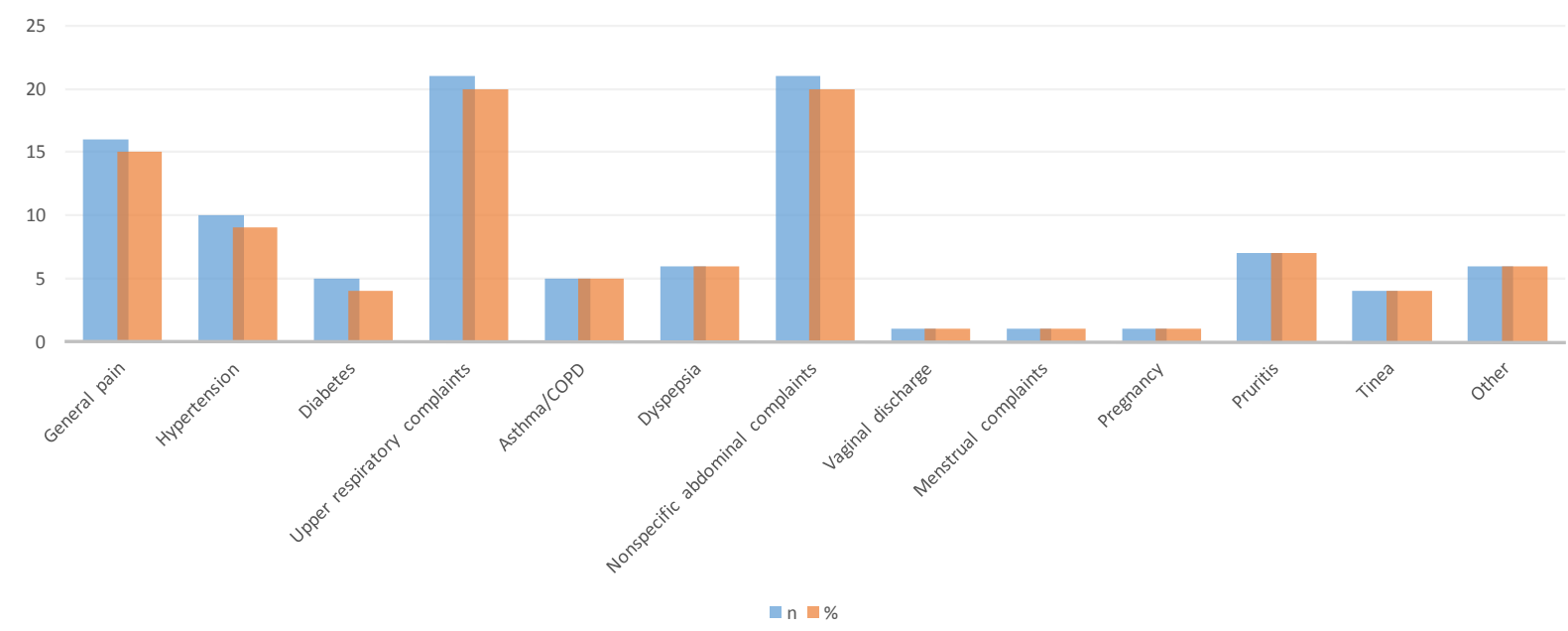

Figure 4. Diagnoses made by clinicians during two days of primary care brigade clinics near Limon, Honduras.

dyspepsia, and nonspecific abdominal complaints were the most commonly reported syndromes. The tracked case definitions cumulatively made up 94\% of all diagnoses, which is similar to the results reported in other Latin American primary care MST settings [6]. This suggests that sending organizations should have a standard approach and appropriate resources for the treatment of these common symptoms.

This study was unique in its use of standardized case definitions. These definitions replaced diagnostic schema that would rely on clinicians to make pathologic and microbiological diagnoses such as parasitic infection, giardiasis, peptic ulcer disease, or bacterial vaginosis. Given the inability to objectively confirm many of these presumptive diagnoses, inter-clinician variability would likely be high. We believe these broader symptombased case definitions to be more precise for epidemiologic surveillance [19]. The use of care definitions could also facilitate the syndromic management of patients seen on MSTs, and improve the consistency of patience care offered by MSTs. Further study is required to ensure that such case definitions remain appropriate and reliable across varied settings.

This pilot study made use of a novel mobile EMR designed for use on mobile MSTs in LMICs. The development of mobile-ready electronic records holds promise for improving the quality of medical records and continuity of care on MSTs abroad [20] [21]. In Latin America, an internally developed brigade EMR is being used for record keeping on MSTs in Ecuador, Dominican Republic, and Guatemala [11]. In this study, the QuickChart EMR was acceptable for clinical use and documentation of patient diagnoses at the point of care. While a previous study describes use of Palm Pilots to support a MST in Kenya [22], this study is the most recent example of a smartphone-based EMR being used for diagnostic tracking in a primary care mobile MST setting. 
Given the consistency of syndromes encountered on such remote medical trips, it would be useful to automate and standardize prescriptions and protocols for common and important diagnoses. The combination of such decision support with the production of guidelines for such MSTs would help minimize variability in practice between clinicians and improve quality of patient care [23]-[25]." Furthermore, the ability for clinicians to electronically review relevant past medical history concerning chronic conditions (hypertension, diabetes, epilepsy) would aid in effective decision making in a busy clinic. This type of decision support, however, would ultimately remain on the future development of appropriate evidence based algorithms.

Weaknesses of the study include the small sample size and presence of only one investigator. In keeping with the five developmental stages for a medical informatics intervention described by Stead [26], this early trial satisfied the need to demonstrate viability of the mobile EMR in the field. Furthermore, it provided proof of concept for the prospective viability of case definitions and electronic data entry.

\section{Conclusion}

In this observational pilot study, simple case definitions were used in a resource-limited setting to track epidemiology on a North American-led MST. Such case definitions may allow standardization of diagnoses made by multiple clinicians from different backgrounds, and suggest a basis for the development of evidence-based guidelines for common complaints. The use of mobile EMRs holds promise for planning, needs assessment, and patient follow-up on similar trips.

\section{Authors' Disclaimers}

\section{Acknowledgments}

Carolina Honduras Health.

\section{Funding}

None.

\section{Competing Interests}

The authors are co-developers of the QuickChart EMR mobile electronic medical record for medical service trips.

\section{Ethical Approval}

None required.

\section{References}

[1] Sykes, K.J. (2014) Short Term Medical Service Trips: A Systematic Review of the Literature. American Journal of Public Health, 104, e38-e48. http://dx.doi.org/10.2105/AJPH.2014.301983

[2] Wall, A. (2011) The Context of Ethical Problems in Medical Volunteer Work. HEC Forum, 23, 79-90. http://dx.doi.org/10.1007/s10730-011-9155-8

[3] Badame, A.J. (1988) Incidence of Skin Diseases in Rural Jamaica. International Journal of Dermatology, 27, $109-111$. http://dx.doi.org/10.1111/j.1365-4362.1988.tb01283.x

[4] Mahé, A., Thiam N’Diaye, H. and Bobin, P. (1997) The Proportion of Medical Consultations Motivated by Skin Diseases in The Health Centers of Bamako (Republic of Mali). International Journal of Dermatology, 36, 185-186. http://dx.doi.org/10.1046/j.1365-4362.1997.00140.x

[5] Martiniuk, A.L., Adunuri, N., Negin, J., Tracey, P., Fontecha, C. and Caldwell, P. (2012) Primary Care Provision by Volunteer Medical Brigades in Honduras: A Health Record Review of More than 2500 Patients over Three Years. International Journal of Health Services, 42, 739-753. http://dx.doi.org/10.2190/HS.42.4.i

[6] Dainton, C. and Chu, C.H. (2015) Symptom Clusters on Primary Care Medical Service Trips in Five Regions in Latin America. Journal of Epidemiology and Global Health, 5, 259-264. http://dx.doi.org/10.1016/j.jegh.2014.12.002

[7] Rodríguez-Vera, F.J., Marín, Y., Sánchez, A., Borrachero, C. and Pujol, E. (2002) Illegible Handwriting in Medical Records. Journal of the Royal Society of Medicine, 95, 545-546. http://dx.doi.org/10.1258/jrsm.95.11.545 
[8] Pirkle, C.M., Dumont, A. and Zunzunegui, M. (2012) Medical Recordkeeping, Essential but Overlooked Aspect of Quality of Care in Resource-Limited Settings. International Journal for Quality in Health Care, 24, 564-567. http://dx.doi.org/10.1093/intqhc/mzs034

[9] Fraser, H.S., Blaya, J., Choi, S.S., Bonilla, C. and Jazayeri, D. (2006) Evaluating the Impact and Costs of Deploying an Electronic Medical Record System to Support TB Treatment in Peru. AMIA Annual Symposium Proceedings, 264-268.

[10] Buckler, S.M., Wiliam, F.D., Thomas, C.J. and Mark, B. (2013) Electronic Medical Record iPad Application. Honors Thesis Projects, University of Tennessee, Knoxville. http://trace.tennessee.edu/utk_chanhonoproj/1627

[11] MacGregor, M. and Ahmed, M. (2014) TimmyCare: An Electronic Medical Record for Resource-Poor Environments. The Lancet Global Health, 2, S30. http://dx.doi.org/10.1016/s2214-109x(15)70052-5

[12] Whitworth, J.A. (2003) World Health Organization, International Society of Hypertension Writing Group. World Health Organization (WHO)/International Society of Hypertension (ISH) Statement on Management of Hypertension. Journal of Hypertension, 21, 1983-1992.

[13] World Health Organization (2012) Prevention and Control of Noncommunicable Diseases: Guidelines for Primary Healthcare in Low Resource Settings. World Health Organization, Geneva.

[14] World Health Organization (2005) Epidemiology and Management of Common Skin Diseases in Children in Developing Countries. http://apps.who.int/iris/bitstream/10665/69229/1/WHO_FCH_CAH_05.12_eng.pdf?ua=1\&ua=1

[15] Medicins sans Frontieres (2013) Clinical Guidelines: Diagnosis and Treatment Manual for Curative Programmes in Hospitals and Dispensaries. Guidance for Prescribing. http://refbooks.msf.org/msf docs/en/clinical guide/cg en.pdf

[16] Schwartz, M.D. (2002) Dyspepsia, Peptic Ulcer Disease, and Esophageal Reflux Disease. Western Journal of Medicine, 176, 98-103.

[17] Global Initiative for Chronic Obstructive Lung Disease (2013) Global Strategy for the Diagnosis, Management, and Prevention of Chronic Obstructive Pulmonary Disease. http://www.goldcopd.org/uploads/users/files/GOLD_Report_2013_Feb20.pdf

[18] International Diabetes Foundation Clinical Guidelines Task Force (2006) Global Guideline for Type 2 Diabetes: Recommendations for Standard, Comprehensive, and Minimal Care. Diabetic Medicine, 23, 579-593. http://dx.doi.org/10.1111/j.1464-5491.2006.01918.x

[19] Krause, G., Brodhun, B., Altmann, D., Claus, H. and Benzler, J. (2006) Reliability of Case Definitions for Public Health Surveillance Assessed by Round-Robin Test Methodology. BMC Public Health, 6, 129. http://dx.doi.org/10.1186/1471-2458-6-129

[20] Warren, A.K. (2006) Can the Ubiquitous Power of Mobile Phones Be Used to Improve Health Outcomes in Developing Countries? Globalization and Health, 2, 9. www.globalizationandhealth.com/content/2/1/9 http://dx.doi.org/10.1186/1744-8603-2-9

[21] Kinkade, S. and Verclas, K. (2008) Wireless Technology for Social Change. UN Foundation-Vodafone Group Foundation Partnership, Washington DC and Berkshire.

[22] Merrell, R.C., Merriam, N. and Doarn, C. (2004) Information Support for the Ambulant Health Worker. Telemedicine Journal and e-Health, 10, 432-436. http://dx.doi.org/10.1089/tmj.2004.10.432

[23] Hunt, D.L., Haynes, R.B., Hanna, S.E. and Smith, K. (1998) Effects of Computer-Based Clinical Decision Support Systems on Physician Performance and Patient Outcomes: A Systematic Review. JAMA, 280, 1339-1346. http://dx.doi.org/10.1001/jama.280.15.1339

[24] Kawamoto, K., Houlihan, C.A., Balas, E.A. and Lobach, D.F. (2005) Improving Clinical Practice Using Clinical Decision Support Systems: A Systematic Review of Trials to Identify Features Critical to Success. BMJ, 330, 765. http://dx.doi.org/10.1136/bmj.38398.500764.8F

[25] Middleton, B. (2009) The Clinical Decision Support Consortium. Studies in Health Technology and Informatics, 150, 26-30.

[26] Stead, W.W., Haynes, R.B., Fuller, S., Friedman, C.P., Travis, L.E., Beck, J.R., et al. (1994) Designing Medical Informatics Research and Library-Resource Projects to Increase What Is Learned. Journal of the American Medical Informatics Association, 1, 28-33. http://dx.doi.org/10.1136/jamia.1994.95236134 